trend $<0.001)$ respectively. Addition of ankle-brachial index significantly $(p<0.001)$ increased the predictive value of the model for 3 year deaths compared with a model containing risk factors alone. Comparison of areas under receiver operator characteristics curves confirmed that a model including the ankle-brachial index discriminated better than one without.

Conclusions There was an inverse association between anklebrachial index and mortality. Addition of ankle-brachial index significantly improved the prediction of 3-year mortality over and above that of conventional risk factors. We recommend that anklebrachial index be incorporated into prognostic assessment for patients with ischaemic heart disease.

\section{e0290 LOWER ESTIMATED GLOMERULAR FILTRATION RATE CORRELATES TO ARTERIAL STIFFNESS IN GENERAL CHINESE WOMEN WITH NORMAL RENAL FUNCTION}

doi:10.1136/hrt.2010.208967.290

Bian Suyan, Luo Leiming, Xiao Tiehui, Oi Liping, Wu Hongmei, Xiao Wenkai, Sheng Li, Duan Liufa. Chinese Pla General Hospital

Background and Objective Although extensive research has shown the association of decreased renal function with early arteriosclerosis in patients with chronic kidney disease, it's still controversy whether decreased glomerular filtration rate (GFR) and menopause have independent relationship with arterial stiffness in general women residents with normal renal function. The aim of this study was to identify how decreased GFR or menopause contributes to central and peripheral arterial stiffness.

Methods In this cross-sectional study, we randomly recruited 1,131 women residents (mean age: $56.59 \pm 14.16$ years) with normal GFR levels estimated by the abbreviated Modification of Diet in Renal Disease equation (defined as eGFR $\geq 60 \mathrm{ml} / \mathrm{min} / 1.73 \mathrm{~m}^{2}$ ) from three large communities in Beijing. Arterial stiffness was assessed by measuring carotid-femoral PWV (PWVcf), carotid-radial PWV (PWVcr) and augmentation index (AIx) with validated automatic devices. Menopause status was defined by the cut-off age of 50, the median menopause age of Chinese women.

Results Subjects from the lowest eGFR tertile group had the highest PWVcf and AIx, while the values of PWVcr were comparable. Meanwhile, eGFR was inversely correlated with PWVcf and AIx but not with PWVcr. Multiple stepwise regression demonstrated a significant relationship between eGFR and PWVcf, independent of the conventional atherosclerotic risk factors. This association was not significant between eGFR values and PWVcr or AIx. Lower eGFR accompanied by higher PWVcf and AIx was evident in the post-menopause group while the PWVcr values were comparable. Post-menopause was an independent predictor for PWVcf and AIx, but not for PWVcr.

Conclusions In general Chinese women with normal renal function, decreased eGFR seems to affect the core arteries other than the peripheral ones. Post-menopause might play part role in arterial stiffness.

\section{e0291 EFFECTS OF XINFUKANG ORAL LIQUID ON THE ACTIVITIES OF RESPIRATORY ENZYME IN EXPERIMENTAL CONGESTIVE HEART FAILURE RATS}

doi:10.1136/hrt.2010.208967.291

Weiwe Wang, Xuebin Cao, Shule Xu, Jiancheng He, Yuanhui Hu. Department of Cardiology, 252 Hospital of PLA

Objective To test the effect of Xin Fu Kang Oral Liquid on the activities of respiratory enzyme (I-IV) in pressure overload-induced left ventricular hypertrophy in rats.
Methods The models of congestive heart failure (CHF) were established by constricting the abdominal aorta of rats partly. $75 \mathrm{SD}$ rats were randomly divided into Sham operation $(\mathrm{SH})$, Coarctation of abdominal aorta model group (CAA) and Xinfufang Oral Liquid group (XFK). The activities of respiratory enzyme (I-IV) were respectively measured by spectrophotometric method in every group at the 10th, 12th week after the interventional of the drugs. Results The study shows that CAA group the activities of respiratory enzyme significantly decreased, the activities of respiratory enzyme II (SDH), IV (CCO) have obviously difference ( $p<0.01)$, In the XFK group the activities of respiratory enzyme obviously increased compared with CAA and by the 10th, 12thweek, SDH, CCO have obviously difference $(p<0.01)$, The activities of respiratory enzyme of the 12th week in XFK group obviously increased compared with that of the 10th week, SDH, CCO have obviously difference $(p<0.05)$.

Conclusion XinFuKang Oral Liquid can obviously improve the activities of respiratory enzyme of congestive heart failure rats.

\section{E0292 SURVEY OF CURRENT SMOKING STATUS OF PATIENTS AT CARDIOLOGY CLINICS IN BEIJING}

doi:10.1136/hrt.2010.208967.292

${ }^{1}$ Rong Jing Ding, ${ }^{2}$ Jinming Yu, ${ }^{2}$ Lijun Zhang, ${ }^{1}$ Dayi Hu. ${ }^{1}$ Heart and Vascular Center,

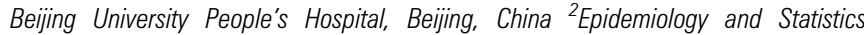
department, Fudan University, ShangHai, China

Objective To understand the current status of smoking patients at cardiology clinics in Beijng municipal Class 3A hospital, so as to target the anti-smoking intervention.

Methods During March 1-10, 2009, the patients presenting at cardiology clinics were consecutively recruited and a questionnaire survey conducted.

Results A total of 816 valid questionnaires were collected. There were 531 males and 336 females with an age range of 22-80 years old. Among these patients, $90.5 \%$ knew the fact that smoking was harmful to cardiovascular health, $67.4 \%$ non-smokers, $15.8 \%$ smokers and $15.8 \%$ quitters. The smoking rate among the patients with coronary heart disease, diabetes mellitus, hyperlipidaemia and hypertension was $28.4 \%, 46.7 \%, 50 \%$ and $73.3 \%$ respectively. The smokers consumed a median of 15 cigarettes per day. $71.8 \%$ of them did not wish to quit. Those with nicotine dependence score under 4 accounted for $64.2 \%$ and above 5 accounted for $35.8 \%$. The quitting urge had no correlation with nicotine dependence score or cardiovascular disease categories.

Conclusion The smoking rate among the patients presenting at cardiology clinic is lower than that of general population. The smokers had a lesser urge to quit. Besides strengthening smokingquitting interventions in coronary heart disease patients an outpatient department, cardiologist should also do so for those smokers with cardiovascular risk factors.

\section{E0293 SURVEY 2009 OF CURRENT SMOKING STATUS OF URBAN AND RURAL RESIDENTS IN BEIJING REGION}

doi:10.1136/hrt.2010.208967.293

${ }^{1}$ Rong Jing Ding, ${ }^{2}$ Jinming Yu, ${ }^{2}$ Lijun Zhang, ${ }^{1}$ Dayi Hu. ${ }^{1}$ Heart and Vascular Center, Beijing University People's Hospital, Beijing, China ${ }^{2} E$ Eidemiology and Statistics Department, Fudan University, ShangHai, China

Objective To understand the smoking rate, distribution characteristics and quitting urge of urban and rural populations in Beijing region in 2009 so as to provide basal materials for future antismoking population interventions. 